\title{
Superior Vena Cava Stenosis
}

In August 2000, a 42-year-old female, presented to the emergency department complaining of fever, night sweating and weight loss. Physical examination showed left inguinal adenopathy of $1 \mathrm{~cm}$. Blood tests were normal. Thoracic computerized tomography (CT) scan showed left pleural effusion and axillary, mediastinal, retroperitoneal and inguinal adenopathies. Biopsy of inguinal adenopathy revealed Non Hodgkin Follicular Lymphoma (CD20+). Tretment with cyclophosphamide, doxorrubicin, vincristine and prednisolone (CHOP) was started. CT scan evaluation after 6 cycles showed persistent mediastinal adenopathies. The patient started mediastinal radiotherapy. In July 2006, a new left pleural effusion and mediastinic adenopathies appeared. The patient started chemotherapy with rituximab, ifosfamide, carboplatin and etoposide (R-ICE) and achieved a complete response. In October 2010, recurrence of the disease was detected. Chemotherapy was restarted and complete response was achieved. In January 2014, the patient presented with facial edema which worsened in the morning (figure 1). She also complained about enlarged mammary veins and dyspnea. Positron emission tomography (PET) scan was negative for revealing recurrence of the disease. Brain CT scan was normal. Thyroid function, cortisol and auto-immune antibodies were negative. The patient started corticotherapy (1mg/kg/day) and hyperbaric oxygen therapy. The symptoms did not disappear. Thoracic angiography showed superior vena cava stenosis and thrombosis (figure 2). The patient started therapy with enoxaparin and therefore was submitted to dilation of the vena cava stenosis; the symptoms disappeared. Follicular lymphoma represents the second most common Non Hodgkin Lymphoma in adults, accounting for $10-20 \%$ of all lymphomas ${ }^{1}$. It is considered an indolent disease characterized by a relapsing pattern over years. Superior vena cava (SVC) syndrome refers to a constellation of symptoms produced by the obstruction of blood flow through the SVC, resulting in dyspnea, facial and upper-extremity edema and chest pain. Malignancies represent $60 \%-85 \%$ of the etiologies ${ }^{2}$ and lymphoma and lung cancer represent $95 \%$ of malignancy-related SVC syndrome. The authors wish to emphasize a late complication due to stenosis of superior vena cava which mimicked a superior vena cava syndrome. The symptoms were assumed to be a consequence of fibrosis and stenosis caused by previous radiotherapy; this condition is described as a rare complication in the literature ${ }^{3}$.

\section{References}

1. Luminari S., Bellei M., Biasoli I., Federico M. Follicular lymphoma - treament and prognostic factors. Rev Bras Hematol Hemoter. 2012; 34(1): 54-59.

2. Grant S., Meykler S., Beach D. Superior vena cava syndrome as an initial presentation of low-grade follicular lymphoma. J Community Support Oncol. 2014 Nov; 12(11):415-7.

3. Van Putten JW., Schlosser NJ, Vujaskovic Z, Leest AH, Groen HJ. Superior vena cava obstruction caused by radiation induced venous fibrosis. Thorax 2000; 55 (3): 245-246.

\section{Diagnóstico:}

\section{Superior Vena Cava Stenosis as a late complication in a patient with follicular lymphoma}

\section{Alexandra Mesquita, Fátima Braga \\ Oncology Department Hospital Pedro Hispano Matosinhos}

Correspondencia: alexandra.mesquita@ulsm.min-saude.pt

Como citar este artículo: Mesquita A, Braga F

Superior Vena Cava Stenosis. Galicia Clin 2017; 78 (1): XX-XX

Recibido: 21/06/2016; Aceptado: 23/09/16
Figure 1. Patient with facial edema
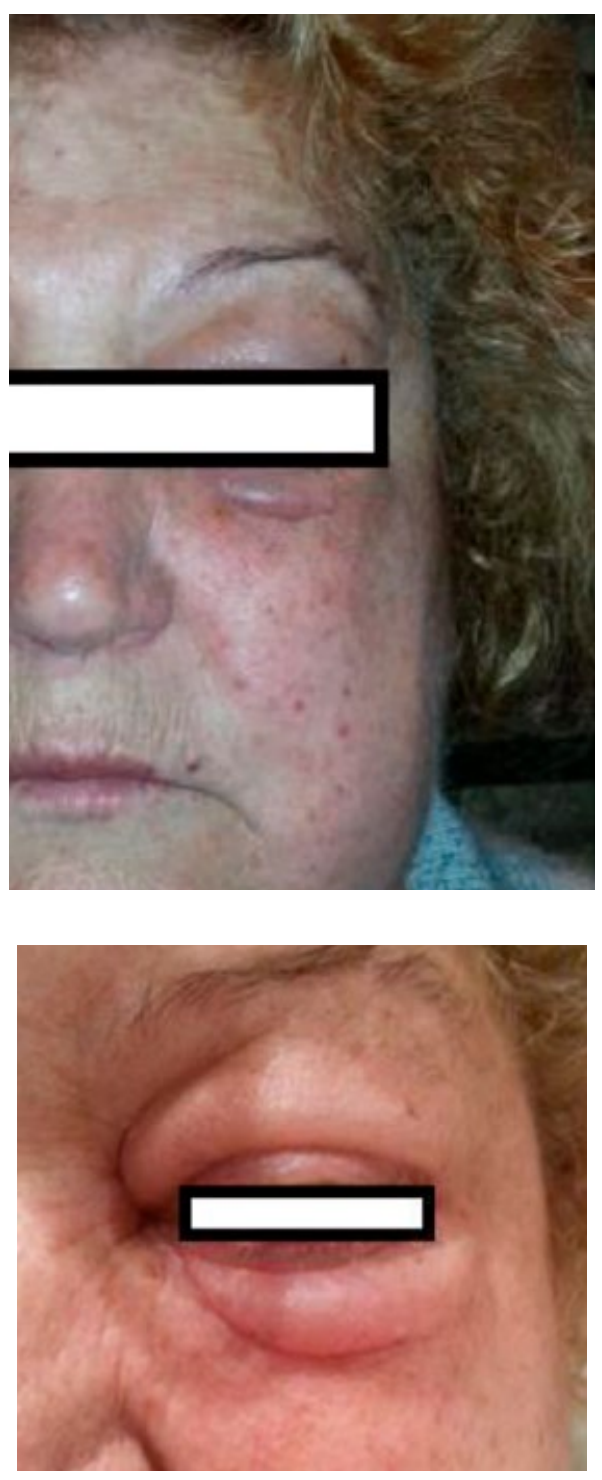

Figure 2. CT Thoracic Angiography

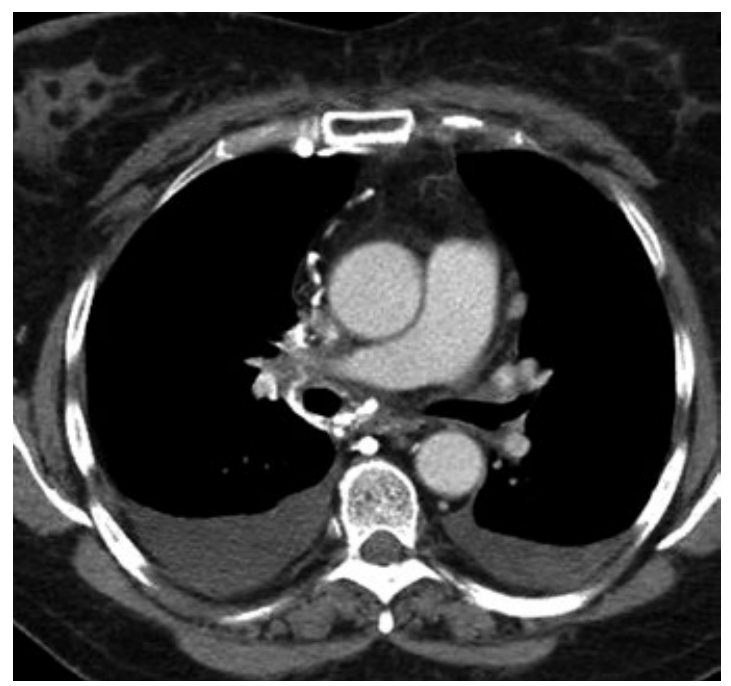

